\title{
ATRIBUTOS FÍSICO-QUÍMICOS DE UM LATOSSOLO DO CERRADO BRASILEIRO E SUA RELAÇÃO COM CARACTERÍSTICAS DENDROMÉTRICAS DO EUCALIPTO $^{(1)}$
}

\author{
César Gustavo da Rocha Lima ${ }^{(2)}$, Morel de Passos e Carvalho ${ }^{(3)}$, \\ Karen Cristina Pirola Narimatsu ${ }^{(2)}$, Matheus Gustavo da Silva ${ }^{(2)} \&$ \\ Hernandez Andrade de Queiroz ${ }^{(2)}$
}

\begin{abstract}
RESUMO
A resistência mecânica à penetração do solo exerce grande influência sobre o crescimento e desenvolvimento vegetal, uma vez que o crescimento das raízes e o rendimento das culturas variam de forma inversamente proporcional ao seu valor. Por outro lado, a matéria orgânica e o pH do solo também são atributos relevantes ao crescimento das plantas, pois estão diretamente ligados à disponibilidade de nutrientes do solo. No ano agrícola 2005/2006, na Fazenda Experimental de Ensino e Pesquisa da Faculdade de Engenharia/UNESP, município de Selvíria, MS $\left(22^{\circ} 23^{\text {, }}\right.$ de latitude S; $51^{\circ} 27^{\prime}$ de longitude W), em um cultivo de Eucalyptus camaldulensis, foram analisados: (a) características dendrométricas da planta: volume de madeira (VOL), perímetro à altura do peito (PAP) e altura (ALT); e (b) atributos do solo em profundidade: resistência mecânica à penetração $(\mathrm{RP})$, umidade gravimétrica (UG), matéria orgânica (MO) e o pH, num Latossolo Vermelho distrófico do Cerrado brasileiro. O objetivo foi estudar as correlações lineares e espaciais entre essas variáveis, buscando determinar indicadores de qualidade do solo para o eucalipto. Dados do solo e da planta foram coletados em uma malha geoestatística com 122 pontos amostrais, numa área de 1,98 ha. Os atributos da planta apresentaram média e alta variabilidade dos dados, ao passo que os do solo mostraram variabilidade baixa, média e alta. Os atributos VOL, PAP, ALT, RP, UG, MO e o pH não variaram aleatoriamente, seguindo padrões espaciais bem definidos, com alcances entre 17 e $169 \mathrm{~m}$. As correlações lineares simples entre as características
\end{abstract}

\footnotetext{
(1) Trabalho realizado na disciplina de Manejo e Conservação do Solo do Programa de Pós-Graduação em Sistemas de Produção, Faculdade de Engenharia, Universidade Estadual Paulista - UNESP. Recebido para publicação em novembro de 2008 e aprovado em outubro de 2009.

(2) Pós-Graduando da Faculdade de Engenharia, Universidade Estadual Paulista - UNESP. Campus de Ilha Solteira. Caixa Postal 31, Av. Brasil, 51, CEP 15385-000 Ilha Solteira (SP). E-mail: etaugustus@yahoo.com.br

(3) Professor Adjunto, Departamento de Fitossanidade, Engenharia Rural e Solos, Faculdade de Engenharia, UNESP. E-mails: morel@agr.feis.unesp.br; mpcarva53@yahoo.com.br
} 
da planta e os atributos do solo foram baixas, porém significativas entre os pares VOL vs RP1, VOL vs RP5, VOL vs MO2 e VOL vs pH1. Do ponto de vista espacial, ocorreu significativa variação inversa do VOL com a RP5 e o pH1. O pH do solo, amostrado de $\mathbf{0}-\mathbf{0 , 1 5} \mathrm{m}$ de profundidade, quando destinado à estimativa da produtividade de madeira do Eucalyptus camaldulensis, apresentou-se como potente indicador da qualidade do solo estudado de Selvíria.

Termos de indexação: resistência mecânica à penetração, propriedades físicas do solo, propriedades químicas do solo, indicadores de qualidade do solo, Eucalyptus camaldulensis.

\title{
SUMMARY: DENDROMETRIC PARAMETERS OF EUCALYPTUS AND THEIR RELATIONSHIP WITH PHYSICAL-CHEMICAL CHARACTERISTICS OF A LATOSOL IN THE BRAZILIAN CERRADO
}

\begin{abstract}
Soil mechanical penetration resistance exerts a strong influence on plant development since the root growth as well as crop yield are inversely related to this variable. On the other hand, soil organic matter and $\mathrm{pH}$ are also relevant for plant development, for being directly linked to soil nutrient availability. In the 2005/2006 growing season, the following dendrometric parameters of Eucalyptus camaldulensis were analyzed: (a) wood volume (WV), (b) perimeter at breast height $(P B H)$ and c) plant height $(P H)$, and the soil attributes: a) mechanical penetration resistance $(M P), b)$ gravimetric moisture $(G R)$, (c) organic matters $(O M)$ and (d) and pH in a Dystrophic Red Latosol of the Cerrado (savanna-like vegetation). The purpose was to study the linear and spatial correlations among these variables, and to obtain soil quality indices for eucalyptus. A geostatistical grid was installed to collect soil and plant data, with 122 sample points, in an area of $1.98 \mathrm{ha}$. The variability of the plant data was medium and high, while the variability of the soil data was low, medium and high. The attributes $W V, P B, P H, M P, G R, O M$, and $p H$ did not change randomly. Rather, they followed well-defined spatial patterns, with ranges between 17 and $169 \mathrm{~m}$. The simple linear correlations among the attributes (plant and soil) were low, however significant for the attribute pairs WV vs $M P 1, W V$ vs $M P 5, W V$ vs $O M 2$ and $W V$ vs $p H 1$. From the spatial correlation viewpoint $W V$ varied significantly, inversely proportional to MP5 and $p H 1$. The soil $p H$, sampled from the 0-0.15 m layer (pH1), turned out to be the best quality indicator of the studied soil toward estimating eucalyptus wood yield.
\end{abstract}

Index terms: mechanical penetration resistance, soil physical properties, soil chemical properties, soil quality indicators, Eucalyptus camaldulensis.

\section{INTRODUÇÃO}

Originário da Austrália, o Eucalyptus spp. foi introduzido no Brasil em 1904, sendo hoje uma das essências florestais que mais têm atendido às necessidades de reposição de matéria-prima no processo industrial, principalmente do setor de papel e celulose (Dossa et al., 2002; IPEF, 2009). Atualmente, as plantações de eucalipto ocupam, no Brasil, uma área de mais de 3,5 milhões de hectares, sendo produzidos comercialmente o volume de até $45 \mathrm{~m}^{3} \mathrm{ha}^{-1} \mathrm{ano}^{-1}$ de madeira, a partir de clones híbridos das espécies mais cultivadas (Mora \& Garcia, 2000; Rapassi et al., 2008). O Eucalyptus camaldulensis, por seu potencial e diversidade de uso, constitui uma espécie de grande interesse econômico, sendo uma das primeiras a serem plantadas com sucesso em regiões fora do continente de origem (FAO, 1979). A literatura tem indicado que suas principais características são dadas pela capacidade de crescer bem em solos relativamente pobres (espécie frugal), resistência a longos períodos de seca, tolerância ao excesso de chuvas, resistência à geada e produção de madeira dura, pesada e escura, quando comparada às espécies E. grandis e E. globulus (Martins et al., 2002). Contudo, o seu crescimento pode ser fortemente alterado por algumas características físico-químicas do solo, principalmente nas condições do Cerrado brasileiro, que, entre outros fatores, comumente é pobre em nutrientes e com elevada acidez.

Entre os atributos físicos do solo de influência direta sobre o crescimento das plantas, pode-se destacar a 
resistência mecânica à penetração. De acordo com Pedrotti et al. (2001), a qualidade física do solo para o crescimento das plantas pode ser determinada pelo grau de resistência mecânica que o solo exerce ao crescimento das raízes, uma vez que, em condições adversas, limita a elongação radicular e, consequentemente, a redução da produtividade vegetal. Em sua avaliação, os dados de resistência mecânica devem ser analisados juntamente aos de umidade do solo, possibilitando assim uma interpretação coerente dos resultados. Dessa forma, pesquisas permitiram concluir que há uma interdependência entre esses atributos, como, por exemplo: (a) a resistência do solo à penetração varia inversamente com a umidade (Tormena et al., 1998); (b) a resistência varia positivamente com a densidade do solo (Imhoff et al., 2000); e (c) a interação desses atributos físicos determina a qualidade do solo (Granatstein \& Bezdicek, 1992).

Os níveis críticos de resistência mecânica à penetração $(\mathrm{RP})$ variam com o tipo de solo e com a espécie cultivada. Entretanto, quando a coleta dos dados de RP é realizada no momento em que a umidade do solo equivale aproximadamente a 2/3 da sua microporosidade (solo friável), inúmeros trabalhos, como os de Santos et al. (2005) e Freddi et al. (2006), têm adotado a seguinte classificação de Arshad et al. (1996): (a) extremamente baixa: $\mathrm{RP}<0,01 \mathrm{MPa}$; (b) muito baixa: $0,01 \leq \mathrm{RP}<0,1 \mathrm{MPa}$; (c) baixa: $0,1 \leq \mathrm{RP}<1,0 \mathrm{MPa} ;$ (d) moderada: $1,0 \leq \mathrm{RP}$ $<2,0 \mathrm{MPa}$; (e) alta: $2,0 \leq \mathrm{RP}<4,0 \mathrm{MPa}$; (f) muito alta: $4,0 \leq \mathrm{RP}<8,0 \mathrm{MPa}$; e (g) extremamente alta: $\mathrm{RP}>8,0 \mathrm{MPa}$.

Dos atributos químicos do solo que exercem grande influência no crescimento e desenvolvimento das plantas podem-se destacar a matéria orgânica e o pH, uma vez que são relativamente de fácil determinação e estão diretamente ligados à disponibilidade de nutrientes do solo (Canellas et al., 1999; Souza et al., 2007). Ainda, ressalta-se que matéria orgânica pode alterar algumas propriedades físicas do solo, como sua agregação, densidade e porosidade, aeração, capacidade de retenção e infiltração de água, podendo prejudicar indiretamente o crescimento vegetal (Raij, 1991; Meurer et al., 2006).

No âmbito da análise de dados, atualmente, a geoestatística também tem sido muito utilizada como ferramenta no estudo da variabilidade dos atributos do solo, mostrando-se importante na interpretação de resultados com base na estrutura da sua variabilidade espacial natural, principalmente da interação entre os atributos físico-químicos do solo com o crescimento vegetal (Araújo, 2002). Nos últimos anos, tem-se verificado substancial aumento de trabalhos com esse objetivo, sobretudo aqueles envolvendo a resistência mecânica à penetração, a matéria orgânica e o $\mathrm{pH}$ do solo (Carvalho et al., 2002, 2003; Souza et al., 2004; Santos et al., 2005; Veronese Júnior et al., 2006; Lima, 2007; Bezerra, 2008; Siqueira et al., 2008).
Diante do exposto, a presente pesquisa objetivou: analisar a variabilidade espacial de atributos do solo e da planta; analisar as correlações lineares e espaciais entre características do crescimento da planta e do solo; e identificar, entre os atributos do solo estudados, aquele de melhor interação linear e espacial com a produtividade de madeira para a área de estudo, os quais possam ser tomados como atributos indicadores da qualidade do solo.

\section{MATERIAL E MÉTODOS}

O experimento foi desenvolvido no ano agrícola de 2005/2006, na Fazenda de Ensino, Pesquisa e Extensão da Faculdade de Engenharia de Ilha Solteira UNESP, localizada no município de Selvíria - MS (latitude $22^{\circ} 23^{\prime}$ S e longitude $51^{\circ} 27^{\prime} \mathrm{W}$ ), com precipitação pluvial média anual de $1.300 \mathrm{~mm}$ e temperatura média de $23,7^{\circ} \mathrm{C}$. O tipo climático é $\mathrm{A}_{\mathrm{w}}$, segundo a classificação de Köppen, caracterizado como tropical úmido com estação chuvosa no verão e seca no inverno (Demattê, 1980). O solo, classificado segundo o Sistema Brasileiro de Classificação de Solos (Embrapa, 2006), é um Latossolo Vermelho distrófico típico argiloso A moderado mesoeutrófico álico caulinítico, mesoférrico muito profundo e fortemente ácido.

A planta-teste foi o Eucalyptus camaldulensis, cujas sementes foram disponibilizadas pelo banco de germoplasma do Centro Nacional de Pesquisa de Florestas (CNPF-EMBRAPA). O talhão (90 x $220 \mathrm{~m})$ foi instalado em 26/04/1986, com espaçamento de $4 \times 4$ m entre plantas, correspondendo a uma densidade populacional de 625 plantas ha-1.

Foram definidas as direções dos eixos cartesianos da rede geoestatística experimental, sendo esta constituída de sete transeções paralelas ao eixo $\mathrm{x}$, contendo 12 pontos de amostragem cada, com um total de 84 pontos, espaçados de $20 \times 15$ m, numa área total de $19.800 \mathrm{~m}^{2}(220 \times 90 \mathrm{~m})$. Também foram alocadas por sorteio duas pequenas malhas de pontos dentro da grande malha, visando detalhar o estudo da dependência espacial para as condições menores do que $15 \mathrm{e}$, ou, $20 \mathrm{~m}$, que ficaram estabelecidas aleatoriamente, entre duas linhas e duas colunas, com um total de 38 pontos amostrais espaçados de $4 \mathrm{~m}$. O total de pontos da malha foi de 122 .

Os atributos do solo e da planta foram individualmente coletados no entorno de cada ponto amostral. Os do solo foram a resistência mecânica à penetração (RP) e a umidade gravimétrica (UG), coletados nas profundidades de $0-0,10,0,10-0,20$, 0,20-0,30, 0,30-0,40 e 0,40-0,50 m; e a matéria orgânica (MO) e o pH, coletados nas profundidades de 0-0,15 e 0,15-0,30 m. A resistência mecânica foi avaliada com o penetrômetro de impactos (Stolf, 1991) e calculada segundo a expressão:

$$
\mathrm{RP}=\{\{5,581+6,891\{[\mathrm{~N} /(\mathrm{P}-\mathrm{A})] 10\}\} 0,0981
$$


em que $\mathrm{RP}$ é a resistência mecânica do solo à penetração $(\mathrm{MPa}) ; \mathrm{N}$, o número de impactos efetuados com o martelo do penetrômetro para obtenção da leitura; e A e P, as leituras antes e depois da realização dos impactos $(\mathrm{cm})$.

As amostras deformadas do solo, para determinação da umidade gravimétrica conforme Embrapa (1997), foram coletadas no mesmo momento que o da resistência mecânica, com trado de caneca. $\mathrm{O}$ pH foi determinado potenciometricamente em solução de $\mathrm{CaCl}_{2}$ 0,01 mol L-1, e o $\mathrm{C}$ orgânico, pelo método da combustão úmida, via colorimétrica, sendo o teor de matéria orgânica do solo calculado pela seguinte expressão (Raij et al., 1987):

$$
\mathrm{MO}=\mathrm{C} .17,24
$$

em que MO é o teor de matéria orgânica $\left(\mathrm{g} \mathrm{dm}^{-3}\right)$ e C é o teor de carbono $\left(\mathrm{g} \mathrm{dm}^{-3}\right)$. Todas as análises foram realizadas no Laboratório de Física e Química do Solo da Faculdade de Engenharia de Ilha Solteira UNESP.

Os atributos da planta avaliados no campo foram o perímetro à altura do peito, em centímetros (PAP), e a altura da árvore, em metros (ALT). Os aparelhos utilizados foram, respectivamente, uma fita métrica e um dendrômetro vertex. Dessa forma, o volume médio de madeira, calculado para cada árvore representante do ponto amostral, foi dado por:

$$
\mathrm{VOL}=1,243410^{-4}\left(\mathrm{PAP}^{2}\right) \mathrm{ALT}
$$

em que VOL é o volume médio anual de madeira $\left(\mathrm{m}^{3} \mathrm{ha}^{-1}\right.$ ano $\left.^{-1}\right)$; PAP é o perímetro médio à altura do peito $(\mathrm{cm})$; ALT, a altura média da árvore $(\mathrm{m})$; e $1,2434.10^{-4}$ foi a constante de ajuste dada pelo quociente $\left[(625.0,5.0,05) /\left(4 \pi .10^{4}\right)\right]$, sendo: (a) 625 foi para transformar o volume de madeira por árvore em volume por ha; (b) $4 \pi$, para transformar raio em diâmetro; (c) $10^{4}$, para transformar $\mathrm{cm}^{2}$ em m²; (d) 0,5, o fator de forma do Eucalyptus camaldulensis; e (e) 0,05, para transformar $\mathrm{m}^{3} \mathrm{ha}^{-1} \mathrm{em} \mathrm{m}^{3} \mathrm{ha}^{-1} \mathrm{ano}^{-1}$.

Para cada atributo estudado, efetuou-se a análise descritiva clássica, com auxílio do software estatístico SAS (Schlotzhaver \& Littell, 1997). Para testar a hipótese de normalidade, ou de lognormalidade, realizou-se o teste de Shapiro \& Wilk (1965) a $1 \%$. Determinou-se uma matriz de correlação linear simples, objetivando efetuar regressões para as combinações, duas a duas, entre os atributos da planta e do solo. Também foi testada a regressão múltipla entre as variáveis dependentes (planta) vs todas as independentes (solo).

A análise geoestatística foi realizada pelo uso do Gamma Design Software 7.0 (GS+, 2004). Aos atributos que apresentaram tendência nos seus dados foi aplicada a técnica do refinamento pela mediana (Silva et al., 2003). Foram ajustados modelos de semivariogramas a todos os atributos estudados e semivariogramas cruzados entre os atributos da planta e do solo, com o objetivo de definir qual atributo tido como variável secundária apresentou melhor correlação espacial explicativa do atributo tido como variável primária. A escolha dos ajustes dos semivariogramas (simples e cruzados) considerou: (a) a menor soma dos quadrados dos desvios (RSS); (b) o maior coeficiente de determinação $\left(\mathrm{r}^{2}\right)$; e (c) o maior avaliador do grau da dependência espacial (ADE). O avaliador do grau da dependência espacial (ADE) foi calculado pela expressão (Lima et al., 2007):

$$
\mathrm{ADE}=[\mathrm{C} /(\mathrm{C}+\mathrm{Co})] 100
$$

em que ADE é o avaliador da dependência espacial; $\mathrm{C}$ é a variância estrutural; e C + Co é o patamar. Dessa forma, a interpretação proposta para o ADE foi a seguinte: $\mathrm{ADE} \leq 25 \%$ indica variável espacial fracamente dependente; $25 \%<\mathrm{ADE} \leq 75 \%$ indica variável espacial moderadamente dependente; e $\mathrm{ADE}>75 \%$ indica variável espacial fortemente dependente.

A escolha do modelo ajustado foi feita por meio da validação cruzada, assim como a definição do tamanho da vizinhança que proporcionou a melhor rede de krigagem e, ou, cokrigagem. A validação cruzada é uma ferramenta que avalia modelos alternativos de semivariogramas simples e cruzados, que efetuarão, respectivamente, a krigagem e a cokrigagem (Vieira, 2000). Assim, a partir do número ideal de vizinhos, foram obtidos os mapas de cokrigagem - resultados da análise da correlação espacial entre os atributos pesquisados da planta e do solo.

Para atributos que apresentaram distribuição lognormal, procedeu-se à retrotransformação pela seguinte expressão (Deutsch \& Journel, 1997):

$$
Z *(x)=10\left[Y^{\left[*(x)+{\frac{\sigma_{k}^{2}}{2}}^{(x)}\right]}\right.
$$

em que $Z^{*}$ (x) é o valor retrotransformado da variável normalizada; $\mathrm{Y}^{*}$ (x) é o valor krigado do logaritmo da variável; e $\frac{\sigma_{k}^{2(x)}}{2}$ é a variância amostral da krigagem do logaritmo da variável. $\mathrm{Na}$ apresentação dos resultados, esses atributos foram discriminados como ' $l$-ATR'.

Quanto aos atributos que apresentaram tendência espacial, os semivariogramas foram determinados, após a retirada da tendência, com os valores dos resíduos (Vieira, 2000). Na apresentação dos resultados, esses atributos foram discriminados como 'r-ATR'.

\section{RESULTADOS E DISCUSSÃO}

Segundo as classes de variabilidade do coeficiente de variação estabelecidas por Pimentel-Gomes (2002), o volume de madeira (VOL) apresentou alta 
variabilidade (30,3\%) enquanto o perímetro à altura do peito (PAP) e a altura (ALT) apresentaram média variabilidade, sendo, respectivamente, 11,8 e $12,0 \%$ (Quadro 1). Para os dados de resistência mecânica à penetração (RP), foi observada magnitude de variabilidade entre baixa e média (9,7-18,9\%), diferentemente do encontrado nos trabalhos de Souza et al. (2001) e Carvalho et al. (2002), que apresentaram dados com variabilidade alta e muito alta. A umidade gravimétrica do solo (UG) apresentou, de modo geral, uma média variabilidade para todos os atributos, à exceção da UG5, que foi baixa. Esses resultados corroboraram os observados por Veronese Júnior et al. (2006). Já a matéria orgânica do solo mostrou média variabilidade, concordando com Souza et al. (1999), ao passo que o pH do solo apresentou variabilidade de baixa magnitude, ficando muito semelhante ao apresentado por Souza et al. (2004).

De acordo com os resultados de Dossa et al. (2002) e Ferreira et al. (2008), o valor médio encontrado no presente estudo, para o volume de madeira $(\mathrm{VOL}=$ $17,9 \mathrm{~m}^{3} \mathrm{ha}^{-1} \mathrm{ano}^{-1}$ ), ficou bem abaixo da produtividade média brasileira de eucaliptos $\left(35,0 \mathrm{~m}^{3} \mathrm{ha}^{-1} \mathrm{ano}^{-1}\right) \mathrm{e}$ muito aquém da produtividade de $60,0 \mathrm{~m}^{3} \mathrm{ha}^{-1} \mathrm{ano}^{-1}$ encontrada para alguns clones de eucalipto das espécies mais plantadas no Brasil, como, por exemplo, o E. grandis e o E. saligna (Pereira et al., 2000). Contudo, os valores médios do PAP $(78,0 \mathrm{~cm}$, equivalente ao diâmetro à altura do peito de $24,8 \mathrm{~cm}$ ) e da ALT $(23,2 \mathrm{~m})$ ficaram próximos daqueles apresentados por Andrade (1961), que relatou para o Eucalyptus camaldulensis, em condições semelhantes às do presente estudo, valores do diâmetro à altura do peito de 27,0 cm e da altura de 18,25 m (Quadro 1).

De acordo com a classificação da resistência mecânica à penetração (Arshad et al., 1996), os valores médios observados (Quadro 1) enquadraram-se na faixa de alta resistência à penetração, com exceção do $l$-RP2 e RP3, que a apresentaram como sendo muito alta. As médias variaram entre 6,4 (l-RP1) e 9,6 $\mathrm{MPa}$ ( $l$-RP2), apresentando, dessa forma, uma tendência não linear dos dados em profundidade no solo, uma vez que, da RP3 até a $l$-RP5, os valores foram menores que o máximo valor, apresentado na segunda camada.

Quadro 1. Análise descritiva inicial de alguns atributos do Eucalyptus camaldulensis e de um Latossolo Vermelho distrófico de Selvíria (MS)

\begin{tabular}{|c|c|c|c|c|c|c|c|c|c|c|}
\hline \multirow{3}{*}{ Atributo $^{(1)}$} & \multicolumn{10}{|c|}{ Medidas estatísticas descritivas } \\
\hline & \multirow[t]{2}{*}{ Média } & \multirow[t]{2}{*}{ Mediana } & \multicolumn{2}{|c|}{ Valor } & \multirow{2}{*}{ Desvio-padrão } & \multicolumn{3}{|c|}{ Coeficiente } & \multicolumn{2}{|c|}{$\begin{array}{c}\text { Probabilidade } \\
\text { do teste } \\
\text { de }\end{array}$} \\
\hline & & & Mínimo & Máximo & & Variação & Curtose & Assimetria & $\operatorname{Pr}<\mathbf{w}$ & DF \\
\hline & & & & & & $\%$ & & & & \\
\hline \multicolumn{11}{|c|}{ Atributos da planta } \\
\hline VOL $\left(\mathrm{m}^{3} \mathrm{ha}^{-1} \mathrm{ano}^{-1}\right)$ & 17,917 & 17,190 & 6,563 & 36,612 & 5,425 & 30,3 & - & - & - & IN \\
\hline $\operatorname{PAP}(\mathrm{cm})$ & 77,977 & 76,993 & 56,529 & 103,040 & 9,219 & 11,8 & 0,052 & 0,037 & 0,284 & NO \\
\hline $\operatorname{ALT}(\mathrm{m})$ & 23,151 & 23,152 & 15,267 & 32,233 & 2,778 & 12,0 & 0,743 & 0,223 & 0,620 & NO \\
\hline \multicolumn{11}{|c|}{ Resistência mecânica do solo à penetração } \\
\hline l-RP1 $(\mathrm{MPa})^{(3,4)}$ & 6,360 & 6,405 & 3,388 & 11,749 & $1,147.10^{-2}$ & 14,3 & $-0,296$ & 0,043 & 0,662 & $\mathrm{LN}$ \\
\hline l-RP2 $(\mathrm{MPa})^{(3,4)}$ & 9,618 & 9,365 & 5,297 & 17,539 & $9,507.10^{-2}$ & 9,7 & 0,371 & 0,374 & 0,091 & $\mathrm{LN}$ \\
\hline RP3 (MPa) & 8,753 & 8,614 & 5,578 & 12,341 & 1,341 & 15,3 & 0,024 & 0,325 & 0,216 & NO \\
\hline RP4 (MPa) & 7,142 & 6,889 & 5,210 & 11,802 & 1,347 & 18,9 & - & - & 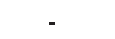 & IN \\
\hline 1-RP5 $(\mathrm{MPa})^{(3,4)}$ & 6,361 & 6,383 & 3,342 & 11,668 & $9,288 \cdot 10^{-2}$ & 11,6 & 0,937 & 0,224 & 0,071 & $\mathrm{LN}$ \\
\hline \multicolumn{11}{|c|}{ Umidade gravimétrica do solo } \\
\hline UG1 (kg kg $\left.{ }^{-1}\right)$ & 0,121 & 0,125 & 0,081 & 0,155 & $1,620.10^{-2}$ & 13,4 & $-0,282$ & $-0,466$ & 0,022 & $\mathrm{TN}$ \\
\hline UG2 $\left(\mathrm{kg} \mathrm{kg}^{-1}\right)$ & 0,124 & 0,128 & 0,088 & 0,154 & $1,562.10^{-2}$ & 12,6 & - & - & - & IN \\
\hline UG3 (kg kg$\left.{ }^{-1}\right)$ & 0,134 & 0,135 & 0,097 & 0,167 & $1,498.10^{-2}$ & 11,2 & $-0,416$ & $-0,192$ & 0,515 & NO \\
\hline UG4 $\left(\mathrm{kg} \mathrm{kg}^{-1}\right)$ & 0,141 & 0,140 & 0,112 & 0,168 & $1,408.10^{-2}$ & 10,0 & - & - & - & IN \\
\hline UG5 $\left(\mathrm{kg} \mathrm{kg}^{-1}\right)$ & 0,146 & 0,147 & 0,115 & 0,178 & $1,396.10^{-2}$ & 9,6 & $-0,821$ & $-0,032$ & 0,039 & $\mathrm{TN}$ \\
\hline \multicolumn{11}{|c|}{ Matéria orgânica do solo } \\
\hline $\operatorname{MO1}\left(\mathrm{g} \mathrm{dm}^{-3}\right)$ & 24,405 & 24,047 & 8,886 & 41,362 & 5,324 & 21,8 & 0,830 & 0,275 & 0,345 & NO \\
\hline $\operatorname{MO} 2\left(\mathrm{~g} \mathrm{dm}^{-3}\right)$ & 13,143 & 12,604 & 3,000 & 24,173 & 3,411 & 26,0 & - & - & - & IN \\
\hline \multicolumn{11}{|c|}{ Reação do solo } \\
\hline pH1 & 4,392 & 4,340 & 4,000 & 5,020 & $2,362 \cdot 10^{-1}$ & 5,4 & - & - & - & IN \\
\hline $\mathrm{pH} 2$ & 4,244 & 4,205 & 3,990 & 4,800 & $1,811.10^{-1}$ & 4,3 & - & - & - & IN \\
\hline
\end{tabular}

(1) VOL: volume de madeira; PAP: perímetro à altura do peito; ALT: altura da árvore; RP e UG, de 1 a 5, são respectivamente a resistência mecânica à penetração e a umidade gravimétrica nas camadas do solo; MO e pH, de 1 a 2 , são respectivamente o teor de matéria orgânica e pH nas camadas do solo. ${ }^{(2)} \mathrm{DF}$ : distribuição de frequência, sendo NO, TN, TL e IN do tipo normal, tendendo a normal, tendendo a lognormal e indeterminado, respectivamente. ${ }^{(3)}$ Valores $\mathrm{x}^{*}$ da média, mediana, mínimo e máximo retrotransformados por $\mathrm{x}^{*}=10^{\log \mathrm{x}}$. 
Entretanto, para a umidade gravimétrica, por apresentar valores crescentes em profundidade no solo, a tendência dos dados foi linear. Os elevados valores de RP foram obtidos principalmente em função dos baixos valores da UG, os quais variaram entre $0,121 \mathrm{~kg} \mathrm{~kg}^{-1}$ (UG1) e $0,146 \mathrm{~kg} \mathrm{~kg}^{-1}$ (UG5). No entanto, o baixo volume médio de madeira encontrado no presente estudo pode ter relação direta com a RP e UG, uma vez que Misra \& Gibbsons (1996) reportaram redução do comprimento das raízes primária e laterais do eucalipto em 71 e $31 \%$, respectivamente com o aumento na $\mathrm{RP}$ do solo de 0,4 a $4,2 \mathrm{MPa}$, muito inferiores às médias encontradas para as diferentes camadas avaliadas do solo.

O valor médio do $\mathrm{pH}$ do solo foi de 4,4 na camada superficial $(0-0,15 \mathrm{~m})$ e de 4,3 na camada subsuperficial $(0,15-0,30 \mathrm{~m})$, indicando, respectivamente, acidez forte e muito forte, conforme Raij (1991), sendo tal característica típica dos solos do Cerrado brasileiro. Por sua vez, o valor médio do teor de matéria orgânica foi de 20,4 e $13,1 \mathrm{~g} \mathrm{dm}^{-3}$, respectivamente para as camadas superficial (MO1) e subsuperficial (MO2), o que, segundo Raij et al. (1997), indica baixo teor de matéria orgânica no solo (Quadro 1).

No quadro 2 são apresentadas as correlações lineares entre as características do Eucalyptus camaldulensis e os atributos do solo. Apesar de todas as correlações serem baixas, fato justificável pelo elevado número de observações $(n=122)$, foi notada significância para as correlações envolvendo $\mathrm{pH}, \mathrm{MO}$, VOL e PAP (Quadro 3).
Tendo em vista o maior interesse agronômico na característica principal da planta (VOL), o destaque ficou por conta dos pares VOL vs $\mathrm{pH} 1 \mathrm{e}$ VOL vs MO2 (Quadro 2), referente às equações (6) e (7) (Quadro 3). Houve variação inversamente proporcional entre causa e efeito, ou seja, com o aumento tanto do $\mathrm{pH}$ quanto da matéria orgânica, o VOL foi reduzido, ao contrário do esperado. No caso do $\mathrm{pH}$, esse fato sugere que essa espécie de eucalipto, como algumas outras, é bastante tolerante à acidez do solo (Vale et al., 1996), visto que os efeitos do baixo $\mathrm{pH}$ (Raij, 1991) não resultaram em limitações, do mesmo modo que o observado por Billet et al. (1988) e Jaiyeoba (1995). Já no caso da MO, a literatura, de forma geral, tem indicado correlação positiva entre MO e crescimento do eucalipto (Grespan, 1997). Do mesmo modo que no presente trabalho, Jonas (2003) identificou significativa correlação negativa $(r=-0,310)$ entre MO e o índice de sítio do eucalipto. No entanto, nada foi relatado ou discutido sobre as possíveis causas dessa interação inesperada.

Pode-se inferir no presente trabalho que a correlação observada (aumento do VOL com a redução dos valores de $\mathrm{pH}$ e MO) não pôde ser explicada por informações da literatura nem por meio dos demais atributos estudados. Provavelmente, para as condições ambientais do local do experimento, houve alguma influência de outros fatores físicos, químicos e, ou, biológicos não medidos. Há de se destacar os pares $\mathrm{PAP}$ vs RP1, PAP vs MO2, PAP vs $\mathrm{pH} 1$, uma vez que foram estatisticamente significativos (Quadro 3). Quanto maiores os valores de RP1, MO e pH1, tanto menor será o PAP.

Quadro 2. Matriz de correlação linear simples entre algumas características do Eucalyptus camaldulensis e de um Latossolo Vermelho distrófico de Selvíria (MS)

\begin{tabular}{|c|c|c|c|c|c|c|c|c|c|c|c|c|c|c|c|c|}
\hline \multirow{2}{*}{$\begin{array}{l}\text { Caracte- } \\
\text { rística }^{(1)}\end{array}$} & \multicolumn{16}{|c|}{ Coeficiente de correlação $^{(2)}$} \\
\hline & VOL & PAP & ALT & $l \mathrm{RP1}$ & $l \mathrm{RP2}$ & RP3 & RP4 & $l$ RP5 & UG1 & UG2 & UG3 & UG4 & UG5 & MO1 & MO2 & PH1 \\
\hline PAP & $0,920^{* *}$ & & & & & & & & & & & & & & & \\
\hline ALT & $0,664^{* *}$ & $0,350^{* *}$ & & & & & & & & & & & & & & \\
\hline$l$-RP1 & $-0,215^{* *}$ & $-0,248^{* *}$ & $-0,046^{\mathrm{ns}}$ & & & & & & & & & & & & & \\
\hline$l$-RP2 & $-0,028^{\text {ns }}$ & $-0,088^{\text {ns }}$ & $0,071^{\mathrm{ns}}$ & $0,492^{* *}$ & & & & & & & & & & & & \\
\hline RP3 & $-0,063^{\mathrm{ns}}$ & $-0,136^{\mathrm{ns}}$ & $0,071^{\text {ns }}$ & $0,185^{* *}$ & $0,558^{* *}$ & & & & & & & & & & & \\
\hline RP4 & $-0,140^{\text {ns }}$ & $-0,151^{*}$ & $-0,116^{\text {ns }}$ & $0,234^{* *}$ & $0,370^{* *}$ & $0,577^{* *}$ & & & & & & & & & & \\
\hline$l$-RP5 & $-0,213^{* *}$ & $-0,200^{* *}$ & $-0,180^{*}$ & $0,232^{* *}$ & $0,292^{* *}$ & $0,312^{* *}$ & $0,662^{* *}$ & & & & & & & & & \\
\hline UG1 & $0,055^{\text {ns }}$ & $0,077^{\mathrm{ns}}$ & $-0,044^{\mathrm{ns}}$ & $-0,365^{* *}$ & $-0,528^{* *}$ & $-0,291^{* *}$ & $-0,150^{*}$ & $-0,086^{\mathrm{ns}}$ & & & & & & & & \\
\hline UG2 & $0,061^{\mathrm{ns}}$ & $0,112^{\mathrm{ns}}$ & $-0,087^{\mathrm{ns}}$ & $-0,341^{* *}$ & $-0,555^{* *}$ & $-0,403^{* *}$ & $-0,255^{* *}$ & $-0,215^{* *}$ & $0,898^{* *}$ & & & & & & & \\
\hline UG3 & $0,115^{\mathrm{ns}}$ & $0,179^{*}$ & $-0,097^{\mathrm{ns}}$ & $-0,275^{* *}$ & $-0,565^{* *}$ & $-0,453^{* *}$ & $-0,370^{* *}$ & $-0,303^{* *}$ & $0,775^{* *}$ & $0,872^{* *}$ & & & & & & \\
\hline UG4 & $0,136^{\mathrm{ns}}$ & $0,194^{* *}$ & $-0,075^{\mathrm{ns}}$ & $-0,281^{* *}$ & $-0,547^{* *}$ & $-0,373^{* *}$ & $-0,371^{* *}$ & $-0,312^{* *}$ & $0,733^{* *}$ & $0,800^{* *}$ & $0,890^{* *}$ & & & & & \\
\hline UG5 & $0,138^{\text {ns }}$ & $0,172^{*}$ & $-0,003^{\mathrm{ns}}$ & $-0,271^{* *}$ & $-0,520^{* *}$ & $-0,326^{* *}$ & $-0,367^{* *}$ & $-0,302^{* *}$ & $0,715^{* *}$ & $0,767^{* *}$ & $0,801^{* *}$ & $0,885^{* *}$ & & & & \\
\hline MO1 & $-0,088^{\mathrm{ns}}$ & $-0,080^{\mathrm{ns}}$ & $-0,110^{\mathrm{ns}}$ & $-0,173^{*}$ & $-0,101^{\text {ns }}$ & $-0,056^{\mathrm{ns}}$ & $0,204^{* *}$ & $0,188^{* *}$ & $0,310^{* *}$ & $0,252^{* *}$ & $0,172^{*}$ & $0,145^{*}$ & $0,118^{\mathrm{ns}}$ & & & \\
\hline $\mathrm{MO} 2$ & $-0,184^{* *}$ & $-0,189^{* *}$ & $-0,067^{\mathrm{ns}}$ & $0,103^{\text {ns }}$ & $0,033^{\text {ns }}$ & $0,066^{\mathrm{ns}}$ & $0,337^{* *}$ & $0,335^{* *}$ & $0,191^{* *}$ & $0,072^{\mathrm{ns}}$ & $-0,015^{\mathrm{ns}}$ & $-0,078^{\mathrm{ns}}$ & $-0,076^{\mathrm{ns}}$ & $0,575^{* *}$ & & \\
\hline pH1 & $-0,225^{* *}$ & $-0,207^{* *}$ & $-0,144^{\mathrm{ns}}$ & $-0,040^{\mathrm{ns}}$ & $-0,117^{\text {ns }}$ & $0,041^{\text {ns }}$ & $0,112^{\mathrm{ns}}$ & $0,187^{*}$ & $0,243^{* *}$ & $0,226^{* *}$ & $0,181^{*}$ & $0,181^{*}$ & $0,209^{* *}$ & $0,482^{* *}$ & $0,230^{* *}$ & \\
\hline $\mathrm{pH} 2$ & $-0,088^{\text {ns }}$ & $-0,166^{*}$ & $-0,001^{\text {ns }}$ & $0,011^{\mathrm{ns}}$ & $0,098^{\text {ns }}$ & $0,084^{\text {ns }}$ & $0,078^{\text {ns }}$ & $0,156^{*}$ & $0,205^{* *}$ & $0,172^{*}$ & $0,128^{\text {ns }}$ & $0,131^{\mathrm{ns}}$ & $0,143^{\mathrm{ns}}$ & $0,396^{* *}$ & $0,245^{* *}$ & $0,634^{* *}$ \\
\hline
\end{tabular}

(1) VOL: volume de madeira, PAP é o perímetro à altura do peito e ALT é a altura da árvore; RP e UG, de 1 a 5, são respectivamente a resistência mecânica à penetração e a umidade gravimétrica nas camadas do solo; $\mathrm{MO}$ e $\mathrm{pH}$, de 1 a 2 , são respectivamente a matéria orgânica e o pH nas camadas do solo; atributos precedidos de "l" foram transformados pelos logaritmos decimais. (2) *: Significativo a $5 \%$; **: Significativo a $1 \%$; ns: Não significativo. 
Quadro 3. Equação de regressão linear simples e coeficientes do ajuste entre algumas características do Eucalyptus camaldulensis e de um Latossolo Vermelho distrófico de Selvíria (MS)

\begin{tabular}{|c|c|c|c|c|}
\hline \multirow{2}{*}{ Modelo matemático(1) } & \multicolumn{3}{|c|}{ Coeficiente de ajuste ${ }^{(2)}$} & \multirow{2}{*}{$\begin{array}{c}\text { Número } \\
\text { eq. }\end{array}$} \\
\hline & $\mathbf{a}$ & $\mathbf{b}$ & $\mathbf{r}$ & \\
\hline \multicolumn{5}{|c|}{ Planta x solo } \\
\hline $\mathrm{VOL}=\mathrm{a} \cdot \exp ^{\mathrm{pH} 1 \cdot \mathrm{b}}$ & $6,028.10^{-1}$ & $-2,863.10^{-1} * *$ & $-0,255^{* *}$ & (6) \\
\hline $\mathrm{VOL}=\mathrm{a} \cdot \mathrm{MO} 2^{\mathrm{b}}$ & 2,757 & $-1,783.10^{-1} * *$ & $-0,184^{* *}$ & (7) \\
\hline $\mathrm{PAP}=\mathrm{a} \cdot \exp ^{\mathrm{RP} 1 \cdot \mathrm{b}}$ & 87,5074 & $-1,8560.10^{-2} * *$ & $-0,24 * * *$ & (8) \\
\hline $\mathrm{PAP}=\mathrm{a} \cdot \exp ^{\mathrm{MO} 2 \cdot \mathrm{b}}$ & $8,437.10^{-1}$ & $-6,520.10^{-1} * *$ & $-0,180^{* *}$ & (9) \\
\hline $\mathrm{PAP}=\mathrm{a} \cdot \exp ^{\mathrm{pH} 1 . b}$ & $1,218.10^{2}$ & $-1,032.10^{-1} * *$ & $-0,207^{* *}$ & (10) \\
\hline $\mathrm{VOL}=\mathrm{a} \cdot \mathrm{PAP}^{\mathrm{b}}$ & $5,4785.10^{-4}$ & $\begin{array}{l}\text { Planta x planta } \\
2,3800^{* *}\end{array}$ & $0,920 * *$ & (11) \\
\hline
\end{tabular}

(1) VOL é o volume de madeira, PAP é o perímetro à altura do peito; RP: resistência mecânica à penetração na camada do solo; $\mathrm{MO}$ e pH são, respectivamente, a matéria orgânica e o pH nas camadas do solo. ${ }^{(2) * *}$ : Significativo a $1 \%$.

Das correlações estabelecidas exclusivamente entre os atributos da planta, foi observada elevada correlação positiva para o par VOL vs PAP. Desta forma, sendo o VOL de maior interesse agronômico e por ser substancialmente mais difícil de ser obtido entre as demais características da planta, pode-se utilizar o ajuste da equação (11, Quadro 3) para estimar o laborioso volume de madeira (VOL) a partir dos dados de rápida e fácil obtenção, que é o PAP. Por outro lado, quando testada a correlação múltipla entre os atributos da planta $v s$ atributos do solo, os resultados não foram estatisticamente significativos.

A análise geoestatística evidenciou que o PAP, ALT, VOL, $l$-RP1, RP3, $l$-RP5, $r$-UG2, $r$-UG3, $r$-UG4, MO1, pH1 e pH2 apresentaram dependência espacial e evidenciaram que as distribuições dos valores dos atributos no espaço não foram aleatórias (Quadro 4), uma vez que suas classes de dependência espacial variaram entre 50,2 (moderada para $l$-RP5) e 90,8\% (alta para VOL), ficando esse fato em consonância com os trabalhos de Souza et al. (2001), Carvalho et al. (2002), Santos et al. (2005) e Veronese Júnior et al. (2006). Houve exceção para $r$ - $l$-RP2, RP4, $r$-UG1, $r$ UG5 e a MO2, que apresentaram efeito pepita puro.

Neste estudo (Quadro 4), pôde-se constatar que $90,8 \%$ da variação espacial do volume de madeira do Eucalyptus camaldulensis foi explicada pela dependência espacial dessa característica, enquanto seu efeito atribuído ao acaso foi de 9,2\%. Foram ajustados modelos semivariográficos dos tipos esférico, exponencial e gaussiano para as diferentes características, indicando que não há um tipo de ajuste mais frequente ou dominante, conforme já havia sido mostrado nos trabalhos de Souza et al. (1999, 2001, 2004), Carvalho et al. (2002), Santos et al. (2005), Veronese Júnior et al. (2006) e Zanão Júnior et al. (2007). Com relação ao alcance da dependência espacial, o VOL, o PAP e a ALT apresentaram-no respectivamente com os valores de 17,2; 67,5 e 99,3 m. Foram encontrados valores entre $85,7 \mathrm{~m}$ (RP3) e $132,3 \mathrm{~m}$ ( $l$-RP1) para a $\mathrm{RP}$, assim como de 97,5 m ( $r$-UG1) e 169,0 m ( $r$-UG4) para a UG. Esses alcances foram similares àqueles observados por Souza et al. (2001), Carvalho et al. (2002), Santos et al. (2005) e Veronese Júnior et al. (2006). Já a matéria orgânica apresentou alcance de 145,1 m (MO1), muito abaixo do encontrado por Zanão Júnior et al. (2007), ao passo que para o $\mathrm{pH}$ foi de 107,3 m (pH1) e 91,4 m (pH2), ficando esses valores muito próximos do encontrado por Souza et al. (2004).

No quadro 5 e na figura 1 são apresentados os resultados do estudo da correlação espacial entre as características da planta e os atributos do solo. As cokrigagens $\mathrm{VOL}=\mathrm{f}(l-\mathrm{RP} 5), \mathrm{VOL}=\mathrm{f}(\mathrm{pH} 1), \mathrm{VOL}=\mathrm{f}(\mathrm{ALT})$ e VOL $=\mathrm{f}(\mathrm{PAP})$ foram as que apresentaram os mais elevados coeficientes de determinação $\left(\mathrm{r}^{2}\right)$, assim como exibiram lags num único quadrante da função de ajuste semiovariográfico, ou seja, quando tal função foi positiva (primeiro quadrante), não houve lags no quadrante inverso (quarto quadrante) e vice-versa (Figura 1b,d,f,h). Assim, esse fato ficou em consonância com os resultados das co-krigagens apresentados por Lima et al. (2007). Os coeficientes de determinação espacial variaram de 0,618 a 0,287, respectivamente para o VOL $=\mathrm{f}(\mathrm{PAP})$ e o VOL $=\mathrm{f}(l$ RP5), ao passo que os alcances ficaram entre 22,1 [VOL $=\mathrm{f}(l-\mathrm{RP} 5)]$ e 36,0 m $[\mathrm{VOL}=\mathrm{f}(\mathrm{ALT})]$. Desse modo, para a presente condição do solo estudado, as significativas dependências espaciais entre as características da planta (variáveis dependentes) e os atributos do solo (independentes) permitiram estimar a distribuição espacial do volume de madeira do Eucalyptus camaldulensis a partir dos atributos do solo $l$-RP5 (Figura 1a,b) e pH1 (Figura 1c,d).

Os resultados obtidos neste estudo mostraram que o VOL apresentou significativa correlação inversa com o $l$-RP1, l-RP5 e pH1 (Quadro 2). Também, o l-RP5 e 
Quadro 4. Parâmetros dos semivariogramas simples ajustados para algumas características do Eucalyptus camaldulensis e de um Latossolo Vermelho distrófico de Selvíria (MS)

\begin{tabular}{|c|c|c|c|c|c|c|c|c|c|}
\hline \multirow[b]{2}{*}{ Atributo $^{(1)}$} & \multicolumn{9}{|c|}{ Parâmetros } \\
\hline & Modelo $^{(2)}$ & $\begin{array}{c}\text { Efeito pepita } \\
\left(\mathrm{C}_{0}\right)\end{array}$ & $\begin{array}{l}\text { Patamar } \\
\left(\mathrm{C}_{0}+\mathrm{C}\right)\end{array}$ & $\begin{array}{l}\text { Variância } \\
\text { estrutural (C) }\end{array}$ & $\begin{array}{c}\text { Alcance }(m) \\
\left(A_{0}\right)\end{array}$ & $\mathbf{r}^{2}$ & $\mathbf{S Q R}^{(3)}$ & $\mathrm{ADE}^{(4)}$ & $\begin{array}{c}\text { Classe de } \\
\text { dependência } \\
\text { espacial }\end{array}$ \\
\hline \multicolumn{10}{|c|}{ Atributos da planta } \\
\hline $\operatorname{VOL}\left(\mathrm{m}^{3} \mathrm{ha}^{-1} \mathrm{ano}^{-1}\right)$ & gau & 2,240 & 24,330 & 22,090 & 17,147 & 0,875 & 17,800 & 90,8 & alta \\
\hline PAP $(\mathrm{cm})$ & $\exp$ & 12,400 & 51,050 & 38,650 & 67,500 & 0,771 & 226,000 & 75,7 & alta \\
\hline $\operatorname{ALT}(\mathrm{m})$ & $\exp$ & $7,600 \cdot 10^{-1}$ & 5,352 & 4,592 & 99,300 & 0,731 & 5,350 & 85,8 & alta \\
\hline \multicolumn{9}{|c|}{ Resistência mecânica do solo à penetracão } & média \\
\hline r-l-RP2 $(\mathrm{MPa})^{(\mathrm{e})}$ & $\begin{array}{l}\text { gau } \\
\text { epp }\end{array}$ & $\begin{array}{l}3,480.10 \\
6,410.10^{-3}\end{array}$ & $\begin{array}{l}7,580 \cdot 10^{-5} \\
6,410 \cdot 10^{-3}\end{array}$ & $4,100.10^{\circ}$ & 132,329 & $\begin{array}{c}0,743 \\
-\end{array}$ & $\begin{array}{c}9,548.10^{\circ} \\
-\end{array}$ & 54,1 & media \\
\hline $\mathrm{RP} 3(\mathrm{MPa})$ & esf & $4,640 . \mathrm{D}^{-1}$ & 1,385 & $9,210.10^{-1}$ & 85,700 & 0,960 & $3,370.10^{-2}$ & 66,5 & média \\
\hline RP4 (MPa) & epp & 1,832 & 1,832 & & & - & & & \\
\hline l-RP5 (MPa) ${ }^{(\mathrm{e})}$ & $\exp$ & $2,965.10^{-3}$ & $5,950 \cdot 10^{-3}$ & $2,985.10^{-3}$ & 124,800 & 0,670 & $3,186.10^{-6}$ & 50,2 & média \\
\hline \multicolumn{10}{|c|}{ Umidade gravimétrica } \\
\hline r-UG1 $\left(\mathrm{kg} \mathrm{kg}^{-1}\right)^{(\mathrm{e})}$ & epp & $8,900 \cdot 10^{-5}$ & $8,900 \cdot 10^{-5}$ & 5 & - & . & . & - & - \\
\hline $\mathrm{r}$-UG2 $\left(\mathrm{kg} \mathrm{kg}^{-1}\right)^{(\mathrm{e})}$ & esf & $1,500.10^{-5}$ & $5,900 \cdot 10^{-5}$ & $4,700.10^{-5}$ & 97,500 & 0,975 & $3,818.10^{-11}$ & 75,0 & alta \\
\hline $\mathrm{r}$-UG3 $\left(\mathrm{kg} \mathrm{kg}^{-1}\right)^{(\mathrm{e})}$ & $\exp$ & $1,200.10^{-5}$ & $6,100.10^{-5}$ & $4,900.10^{-5}$ & 111,600 & 0,812 & $4,103 \cdot 10^{-10}$ & 80,3 & alta \\
\hline $\mathrm{r}-\mathrm{UG} 4\left(\mathrm{~kg} \mathrm{~kg}^{-1}\right)^{(\mathrm{e})}$ & esf & $1,100.10^{-5}$ & $4,800 \cdot 10^{-5}$ & $3,700.10^{-5}$ & 169,000 & 0,729 & $1,657.10^{-10}$ & 76,4 & alta \\
\hline r-UG5 $\left(\mathrm{kg} \mathrm{kg}^{-1}\right)^{(\mathrm{e})}$ & epp & $7,200 \cdot 10^{-5}$ & $7,200 \cdot 10^{-5}$ & & & - & & & \\
\hline \multicolumn{10}{|c|}{ Matéria orgânica do solo } \\
\hline $\operatorname{MO1}\left(\mathrm{g} \mathrm{dm}^{-3}\right)$ & gau & 7,870 & 15,890 & 8,020 & 145,146 & 0,902 & 7,570 & 50,5 & média \\
\hline $\mathrm{MO} 2\left(\mathrm{~g} \mathrm{dm}^{-3}\right)$ & epp & 11,675 & 11,675 & & & - & & & \\
\hline \multicolumn{10}{|c|}{ Reação do solo } \\
\hline $\mathrm{pH} 1$ & esf & $1,946 \cdot 10^{-2}$ & $5,082.10^{-2}$ & $3,136.10^{-2}$ & 107,300 & 0,987 & $9,857.10^{-6}$ & 61,7 & média \\
\hline $\mathrm{pH} 2$ & esf & $1,070.10^{-2}$ & $2,470.10^{-2}$ & $1,400.10^{-2}$ & 91,400 & 0,897 & $2,418.10^{-5}$ & 56,7 & média \\
\hline
\end{tabular}

(1) VOL: volume de madeira; PAP: perímetro à altura do peito; ALT: altura da árvore; RP e UG, de 1 a 5 , são respectivamente a resistência mecânica à penetração e a umidade gravimétrica nas camadas do solo; $\mathrm{MO}$ e $\mathrm{pH}$ de 1 a 2 são respectivamente a matéria orgânica e o pH nas camadas do solo. ${ }^{(2)}$ exp: exponencial, gau.: gaussiano, esf.: esférico e epp.: efeito pepita puro. ${ }^{(3)}$ SQR: soma dos quadrados dos resíduos. ${ }^{(4)} \mathrm{ADE}$ : avaliador da dependência espacial. ${ }^{(5)}$ Atributos precedidos de "l" e "r" tiveram respectivamente valores representados pelos logaritmos decimais e pelos resíduos.

Quadro 5. Parâmetros dos semivariogramas cruzados ajustados entre algumas características do Eucalyptus camaldulensis e de um Latossolo Vermelho distrófico de Selvíria (MS)

\begin{tabular}{|c|c|c|c|c|c|c|c|c|}
\hline \multirow[b]{2}{*}{ Atributo(1) $^{(1)}$} & \multicolumn{8}{|c|}{ Parâmetros } \\
\hline & Modelo(2) $^{(2)}$ & $\begin{array}{c}\text { Efeito } \\
\text { pepita }\left(\mathrm{C}_{0}\right)\end{array}$ & $\begin{array}{c}\text { Patamar } \\
\left(C_{0}+C\right)\end{array}$ & $\begin{array}{c}\text { Variância } \\
\text { estrutural (c) }\end{array}$ & $\begin{array}{c}\operatorname{Alcance}(\mathrm{m}) \\
\left(\mathrm{A}_{0}\right)\end{array}$ & $\mathbf{r}^{2}$ & $\mathbf{S Q R}^{(3)}$ & $\mathrm{ADE}^{(4)}$ \\
\hline \multicolumn{9}{|c|}{$\gamma(\mathrm{h})$ cruzado entre atributos } \\
\hline $\mathrm{VOL}=\mathrm{f}(l-\mathrm{RP} 5)$ & esf & $-8,100.10^{-3}$ & $-7,020.10^{-2}$ & $-6,210.10$ & 22,100 & 0,287 & $2,681 \cdot 10^{-3}$ & 0,885 \\
\hline $\mathrm{VOL}=\mathrm{f}(\mathrm{pH} 1)$ & esf & $-1,000.10^{-4}$ & $-2,752.10^{-1}$ & $-2,751.10$ & 35,300 & 0,560 & $1,890.10^{-2}$ & 0,999 \\
\hline $\mathrm{VOL}=\mathrm{f}(\mathrm{PAP})$ & $\exp$ & 4,570 & 23,030 & 18,460 & 27,900 & 0,618 & $4,000.101$ & 0,802 \\
\hline $\mathrm{VOL}=\mathrm{f}(\mathrm{ALT})$ & $\exp$ & $1,000.10^{-2}$ & 4,814 & 4,804 & 36,000 & 0,519 & 5,160 & 0,998 \\
\hline
\end{tabular}

(1) VOL: volume de madeira; PAP: perímetro à altura do peito; ALT: altura da árvore; $l$-RP5: resistência mecânica à penetração na camada de $0,40-0,50 \mathrm{~m}$ e o $\mathrm{pH} 1$ corresponde ao $\mathrm{pH}$ na camada do solo de $0-0,15 \mathrm{~m} .{ }^{(2)}$ exp: exponencial, esf: esférico. ${ }^{(3)} \mathrm{SQR}$ : soma dos quadrados dos resíduos. ${ }^{(4)} \mathrm{ADE}$ : avaliador da dependência espacial. ${ }^{(5)}$ Atributo precedido de " $l$ " teve seu valor representado pelo logaritmo decimal.

o pH1 (variáveis secundárias) apresentaram apreciáveis ajustes semivariográficos quando cokrigados com o VOL (variável primária) (Quadro 5, Figura 1a,b,c,d). Contudo, houve substancial superioridade do $\mathrm{pH} 1\left(\mathrm{r}^{2}=0,560\right)$ sobre o $l$ - $\mathrm{RP} 5\left(\mathrm{r}^{2}=\right.$ 0,287). Assim, ficou evidenciado que o $\mathrm{pH} 1$ foi o atributo do solo que mostrou a melhor interação com a produtividade do volume de madeira (VOL) do 
(a)

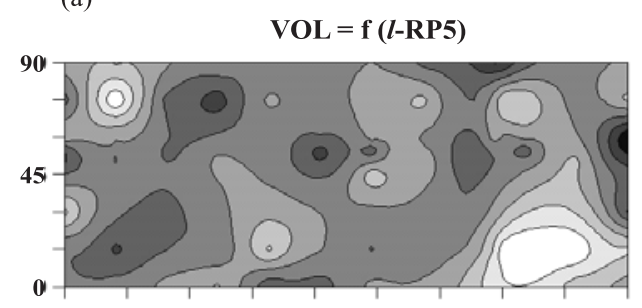

(c)

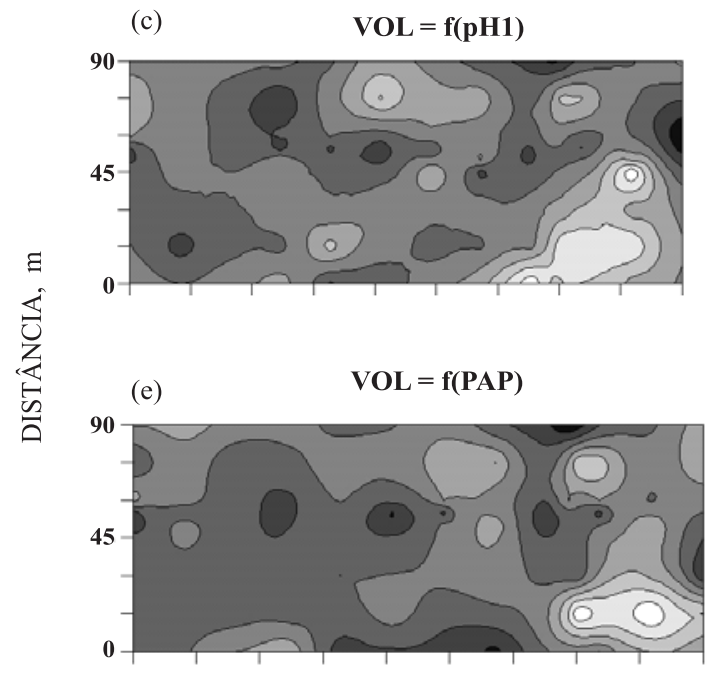

(g)

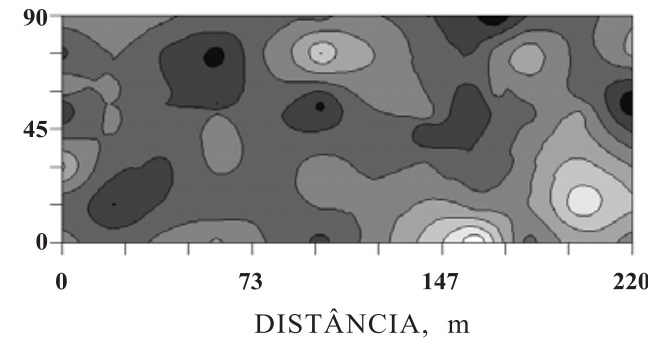

(b) DISTÂNCIA, m

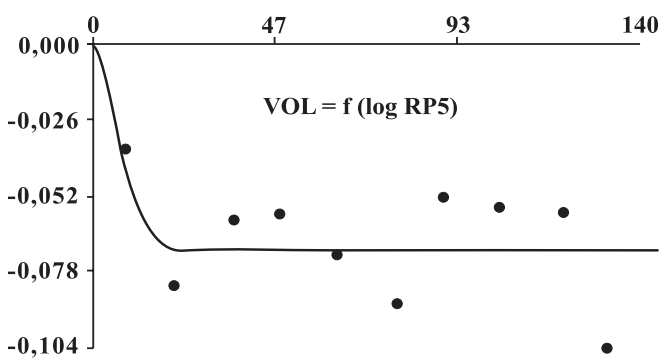

(d)
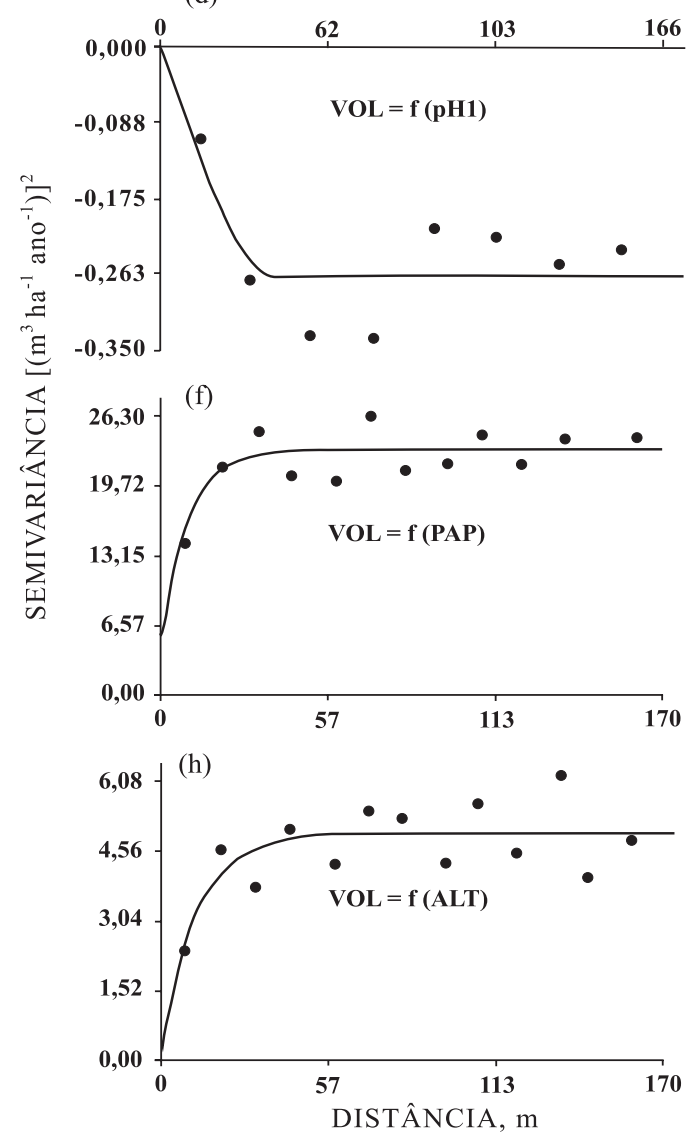

Figura 1. Mapas de cokrigagem e semivariogramas cruzados entre o volume de madeira do Eucalyptus camaldulensis com as características da planta (PAP e ALT) e o l-RP5 e pH1 de um Latossolo Vermelho distrófico de Selvíria (MS).

Eucalyptus camaldulensis para as condições do Cerrado de Selvíria (MS), podendo, portanto, ser tomado como o melhor indicador da qualidade do solo estudado, quando destinado à produtividade de madeira.

\section{CONCLUSÕES}

1. As características da planta mostraram média e alta variabilidade, enquanto para os atributos do solo esta foi baixa, média e alta. Espacialmente, as características da planta e a grande maioria dos atributos do solo apresentaram dependência espacial, seguindo padrões bem definidos.
2. As correlações lineares simples, entre o volume de madeira do Eucalyptus camaldulensis e os atributos do solo, embora baixas, foram significativas quando pareadas com a resistência mecânica à penetração ( $l$ $\mathrm{RP} 1, l$-RP5) e o $\mathrm{pH} 1$.

3. Do ponto de vista espacial, o volume de madeira variou inversamente à resistência mecânica à penetração (l-RP5) e ao $\mathrm{pH}$ do solo (pH1).

4. $\mathrm{O} \mathrm{pH}$ do solo, amostrado de 0 a $0,15 \mathrm{~m}$ de profundidade, quando destinado à estimativa da produtividade de madeira do Eucalyptus camaldulensis, apresentou-se como potente indicador da qualidade do solo estudado de Selvíria. 


\section{LITERATURA CITADA}

ANDRADE, E.N. O Eucalipto. Jundiaí, Cia Paulista de Estradas de Ferro, 1961. 667p.

ARAUJO, A.V. Variabilidade espacial de propriedades químicas e granulométricas do solo na definição de zonas homogêneas de manejo. Jaboticabal, Universidade Estadual Paulista, 2002. 80p. (Tese de Mestrado)

ARSHAD, M.A.; LOWERY, B. \& GROSSMAN, B. Physical tests for monitoring soil quality. In: DORAN, J.W. \& JONES, A.J., eds. Methods for assessing soil quality. Madison, Soil Science Society of America, 1996. p.123141. (SSSA Special Publication, 49)

BEZERRA, C.R.F. Variabilidade espacial da produtividade de forragem do milho safrinha em função de atributos químicos do solo. Ilha Solteira, Universidade Estadual Paulista, 2008. 70p. (Tese de Mestrado)

BILLET, M.F.; FITZPATRICK, E.A. \& CRESSER, M.S. Longterm changes in the acidity of forest soils in North-East Scotland. Soil Use Manag., 4:102-107, 1988.

CANELLAS, L.P.; SANTOS, G.A. \& AMARAL, N.M.B. Reações da matéria orgânica. In: SANTOS, G.A. \& CAMARGO, F.A.O., eds. Fundamentos da matéria orgânica do solo: Ecossistemas tropicais e subtropicais. Porto alegre, Gênesis, 1999. p.69-90.

CARVALHO, M.P.; SORATTO, R.P. \& FREDDI, O.S Variabilidade espacial de atributos físicos em um Latossolo Vermelho distrófico sob preparo convencional em Selvíria (MS). Acta Sci., 24:1353-1361, 2002.

CARVALHO, M.P.; TAKEDA, E.Y. \& FREDDI, O.S Variabilidade espacial de atributos de um solo sob videira em Vitória Brasil (SP). R. Bras. Ci. Solo, 27:695-703, 2003.

DEMATTÊ, J.L.I. Levantamento detalhado de solos do "Campus Experimental de Ilha Solteira". Piracicaba, Escola Superior de Agricultura Luiz de Queiroz, 1980. 44p.

DEUTSCH, C.V. \& JOURNEL, A.G. Geostatistical software library and user's guide, GSLIB. New York, Oxford University, 1997. 363p.

DOSSA, D.; SILVA, H.D.; BELLOTE, A.F.J. \& RODIGHERI, H.R. Produção e rentabilidade do eucalipto em empresas florestais. Colombo, Embrapa, 2002. 4p. (Comunicado Técnico, 83)

EMPRESA BRASILEIRA DE PESQUISA AGROPECUÁRIA EMBRAPA. Serviço Nacional de Levantamento e Conservação do Solo. Manual de métodos de análise de solos. 2.ed. Rio de Janeiro, 1997. 212p.

EMPRESA BRASILEIRA DE PESQUISA AGROPECUÁRIA EMBRAPA. Sistema brasileiro de classificação de solos. 2.ed. Rio de Janeiro, Embrapa, 2006. 306p.

FERREIRA, A.R.; JOÃO, D.M. \& GODOY, L.P. A utilização da matéria-prima eucalipto na indústria moveleira como estratégia para o desenvolvimento sustentável. In: CONGRESSO NACIONAL DE EXCELÊNCIA EM GESTÃO, Niterói, 2008. Anais. Niterói, 2008. CD-ROM.
FOOD AND AGRICULTURE ORGANIZATION - FAO. Eucalypts for planting. Rome, 1979. 677p. (FAO Forestry Series)

FREDDI, O.S.; CARVALHO, M.P.; CARVALHO, G.J. \& VERONESE JUNIOR, V. Produtividade do milho relacionada com a resistência mecânica à penetração do solo sob preparo convencional. Eng. Agric., 26:113-121, 2006 .

GRANATSTEIN, D. \& BEZDICEK, D.F. The need for a soil quality index: Local and regional perspectives. Am. J. Alt. Agric., 7:12-16, 1992.

GRESPAN, S.L. Produção e eficiência nutricional de clones de eucalipto no norte do Espírito Santo e suas relações com características do solo. Viçosa, MG, Universidade Federal de Viçosa, 1987. 81p. (Tese de Mestrado)

GS+: Geostatistics for environmental sciences. 7.ed. Michigan, Plainwell Gamma Desing Software, 2004. 159p.

IMHOFF, S.; SILVA, A.P. \& TORMENA, C.A. Aplicações da curva de resistência no controle da qualidade física de um solo sob pastagem. Pesq. Agropec. Bras., 35:1493-1500, 2000.

INSTITUTO DE PESQUISA E ESTUDOS FLORESTAIS IPEF. Pesquisa florestal no Brasil: Tecnologia de produtos florestais. Disponível em: <http://www.ipef.br/mct/ MCT_04.htm>. Acesso em: 10 abr. 2009.

JAIYEOBA. I.A. Changes in soil properties related to different land uses in part of the Nigerian semi-arid Savannah. Soil Use Manag., 11:84-89, 1995.

JONAS, L.O. Emprego do geoprocessamento no estudo da relação entre potencial produtivo de um povoamento de eucalipto e atributos do solo e do relevo. Piracicaba, Escola Superior de Agricultura Luiz de Queiroz, 2003. 205p. (Tese de Mestrado)

LIMA, C.G.R.; CARVALHO, M.P.; MELLO, L.M.M. \& LIMA, R.C. Correlação linear e espacial entre a produtividade de forragem, a porosidade total e a densidade do solo de Pereira Barreto (SP). R. Bras. Ci. Solo, 31:1233-1244, 2007.

LIMA, R.C. Produtividade de forragem do milho em função de atributos físicos de um Latossolo Vermelho distrófico sob plantio direto. Ilha Solteira, Universidade Estadual Paulista, 2007. 73p. (Tese de Mestrado)

MARTINS, I.S.; PIRES, I.E. \& OLIVEIRA, M.C. Divergência genética em progênies de uma população de Eucalyptus camaldulensis. Flor. Amb., 9:81-89, 2002.

MEURER, E.J.; RHEINHEMEIR, D. \& BISSANI, C.A. Fenômeno de sorção em solos. In: MEURER, E.J., ed. Fundamentos de química do solo. 3.ed. Porto Alegre, Evangraf, 2006. p.117-162.

MISRA, R.K. \& GIBBONS, A.K. Growth and morphology of eucalypt seedling-roots, in relation to soil strength arising from compaction. Plant Soil, 182:1-11, 1996.

MORA, A.L. \& GARCIA, C.H. A cultura do eucalipto no Brasil. São Paulo, Sociedade Brasileira de Silvicultura, 2000. 112p. 
PEDROTTI, A.; PAULETTO, E.A.; CRESTANA, S.; FERREIRA, M.M.; DIAS JUNIOR, M.S.; GOMES, A.S. \& TURATTI, A.L. Resistência mecânica à penetração de um Planossolo submetido a diferentes sistemas de cultivo. R. Bras. Ci. Solo, 25:521-529, 2001.

PEREIRA, J.C.D.; STURION, J.A.; HIGA, A.R.; HIGA, R.C.V. \& SHIMIZU, J.Y. Características da madeira de algumas espécies de eucalipto plantadas no Brasil. Colombo, Embrapa, 2000. 113p.

PIMENTEL-GOMES, F. \& GARCIA, C.H. Estatística aplicada a experimentos agronômicos e florestais: Exposição com exemplos e orientações para uso de aplicativos. Piracicaba, FEALQ, 2002. 309p.

RAIJ, B.van.; CANTARELLA, H.; QUAGGIO, J.A. \& FURLANI, A.M.C., eds. Recomendações de adubação e calagem para o Estado de São Paulo. Campinas, Instituto Agronômico de Campinas, 1997. 285p.

RAIJ, B.van. Fertilidade do solo e adubação. Piracicaba, Ceres, 1991. 343p.

RAIJ, B.van.; QUAGGIO, J.A.; CANTARELLA, H.; FERREIRA, M.E.; LOPES, A.S. \& BATAGLIA, Q.C. Análise química do solo para fins de fertilidade. Campinas, Fundação Cargill, 1987. 170p.

RAPASSI, R.M.A.; TARSITANO, M.A.A.; PEREIRA, J.C.R. \& ARAÚJO, C.A.M. Cultura do eucalipto na região de Suzanápolis, Estado de São Paulo: Análise econômica. Inf. Econ., 38:7-13, 2008.

SANTOS, P.A.; CARVALHO, M.P.; FREDDI, O.S.; KITAMURA, A.E.; FREITAG, E.E. \& VANZELA, L.S. Correlação linear e espacial entre o rendimento de grãos do feijoeiro e a resistência mecânica à penetração em um Latossolo Vermelho distrófico. R. Bras. Ci. Solo, 29:287-295, 2005.

SCHLOTZHAVER, S.D. \& LITTELL, R.C. SAS system for elementary statical analysis. 2.ed. Cary, 1997. 441p.

SHAPIRO, S.S. \& WILK, M.B. An analysys of variance test for normality: Complete samples. Biometrika, 52:591-611, 1965.

SILVA, V.R.; REICHERDT, J.M.; STORCK, L. \& FEIJÓ, S. Variabilidade espacial das características químicas do solo e produtividade de milho em um Latossolo VermelhoAmarelo distrófico arênico. R. Bras. Ci. Solo, 27:1013$1020,2003$.

SIQUEIRA, G.M.; VIEIRA, S.R. \& CEDDIA, M.B. Variabilidade de atributos físicos do solo determinados por métodos diversos. Bragantia, 67:203-211, 2008.
SOUZA, D.M.G.S.; MIRANDA, L.N. \& OLIVEIRA, S.A.V. A acidez do solo e sua correção. In: NOVAIS, R.F.; ALVAREZ V., V.H.; BARROS, N.F.; FONTES, R.L.F.; CANTARUTTI, R.B. \& NEVES, J.C.L., eds. Fertilidade do solo: A acidez do solo e sua correção. Viçosa, MG, Sociedade Brasileira de Ciência do Solo, 2007. p.205-274.

SOUZA, E.G.; JOHANN, J.A.; ROCHA, J.V.; RIBEIRO, S.R.A.; SILVA, M.S.; URIBE-OPAZO, M.A.; MOLIN, J.P.; OLIVEIRA, E.F. \& NÓBREGA, L.H.P. Variabilidade espacial dos atributos químicos do solo em um Latossolo Roxo distrófico da região de Cascavel (PR). Eng. Agríc., 18:80-92, 1999.

SOUZA, Z.M.; MARQUES JÚNIOR, J.; PEREIRA, J.T. \& MOREIRA, L.F. Variabilidade espacial do $\mathrm{pH}, \mathrm{Ca}, \mathrm{Mg}$, e $\mathrm{V} \%$ do solo em diferentes formas do relevo sob cultivo de cana-de-açúcar. Ci. Rural, 34:1763-1771, 2004.

SOUZA, Z.M.; SILVA, M.L.S.; GUIMARÃES, G.L.; CAMPOS, D.T.S.; CARVALHO, M.P. \& PEREIRA, G.T. Variabilidade espacial de atributos físicos em um Latossolo Vermelho distroférrico sob semeadura direta em Selvíria (MS). R. Bras. Ci. Solo, 25:699-707, 2001.

STOLF, R. Teoria e teste experimental de fórmulas de transformação dos dados de penetrômetro de impacto em resistência do solo. R. Bras. Ci. Solo, 15:229-235, 1991.

TORMENA, C.A.; ROLOFF, G. \& SÁ, J.C.M. Propriedades físicas do solo sob plantio direto influenciado por calagem, preparo inicial e tráfego. R. Bras. Ci. Solo, 22:301-309, 1998.

VALE, F.R.; FURTINI NETO, A.E.; RENÓ, N.B.; FERNANDEZ, L.A. \& RESENDE, A.V. Crescimento radicular de espécies florestais em solo ácido. Pesq. Agropec. Bras., 31:609616, 1996

VERONESE JUNIOR, V.; CARVALHO, M.P.; DAFONTE, J.; FREDDI, O.S.; VIDAL VÁZQUEZ, E. \& INGARAMO, O.E. Spatial variability of soil water content and mechanical resistance of Brazilian Ferralsol. Soil Tillage Res., 85:166177,2006

VIEIRA, S.R. Uso de geoestatística em estudos de variabilidade espacial de propriedades do solo. In: NOVAIS, R.F.; ALVAREZ V., V.H. \& SCHAEFER, C.E.G.R., orgs. Tópicos em ciência do solo. Viçosa, MG, Sociedade Brasileira de Ciência do Solo, 2000. v.1. p.1-54.

ZANÃO JÚNIOR, L.A.; LANA, R.M.Q. \& GUIMARÃES, E.C. Variabilidade espacial do $\mathrm{pH}$, teores de matéria orgânica e micronutrientes em profundidades de amostragem num Latossolo Vermelho sob semeadura direta. Ci. Rural, 37:1000-1007, 2007. 
